\title{
UNDERSTANDING THE ROLE OF CONFIGURAL PROCESSING IN FACE EMOTION RECOGNITION IN PARKINSON'S DISEASE
}

\author{
Short title: UNDERSTANDING FACE EMOTION RECOGNITION IN PD \\ Rosanna Cousins ${ }^{* 1}$, Anne Pettigrew ${ }^{1}$, Olivia Ferrie ${ }^{1}$ and J. Richard \\ Hanley $^{2}$
}

${ }^{1}$ Department of Psychology, Liverpool Hope University, Hope Park, LIVERPOOL, L16 9JD

${ }^{2}$ Department of Psychology, University of Essex, Wivenhoe Park, COLCHESTER, CO4 3SQ

*Corresponding author information: Rosanna Cousins, Department of Psychology, Liverpool Hope University, Hope Park, Liverpool, L69 9JD, England.

(email: cousinr@hope.ac.uk).

\section{Abstract:}

This investigation examined whether impairment in configural processing could explain deficits in face emotion recognition in people with Parkinson's disease (PD). Stimuli from the Radboud Faces Database were used to compare recognition of four negative emotion expressions by older adults with PD $(n=16)$ and matched controls $(n=17)$. Participants were tasked with categorising emotional expressions from upright and inverted whole faces and facial composites; it is difficult to derive configural information from these two types of stimuli so featural processing should play a larger than usual role in accurate recognition of emotional expressions. We found that the PD group were impaired relative to controls in recognising anger, disgust and fearful expressions in upright faces. Then, consistent with a configural 
processing deficit, participants with PD showed no composite effect when attempting to identify facial expressions of anger, disgust and fear. A face inversion effect, however, was observed in the performance of all participants in both the whole faces and facial composites tasks. These findings can be explained in terms of a configural processing deficit if it is assumed that the disruption caused by facial composites was specific to configural processing, whereas inversion reduced performance by making it difficult to derive both featural and configural information from faces.

\section{Keywords:}

Composite Faces; Configural processing; Face Emotion Recognition; Face Inversion Effect; Parkinson's disease.

\section{Data availability statement:}

The data that support the findings of this study are available on request from the corresponding author. The data are not publicly available due to privacy or ethical restrictions.

\section{Acknowledgements:}

We are grateful for the support of all our volunteer participants, and also Dr Paul Fitzsimmons, Dr Glynn Scott and UK Parkinson's for their help with recruitment. We dedicate this paper to the memory of Dr Ann DM Davies without whom none of this research would have taken place.

\section{Declarations of Interest:}

None

\section{Funding:}

This research did not receive any specific grant from funding agencies in the public, commercial, or not-for-profit sectors. 


\section{UNDERSTANDING THE ROLE OF CONFIGURAL PROCESSING IN FACE EMOTION RECOGNITION IN PARKINSON'S DISEASE}

\section{Introduction}

Effortless face processing is an evolved skill that is important throughout the lifespan for social competence. The ability we have for rapid recognition of facial identity and emotional expression has triggered much research towards understanding the complexity of face processing mechanisms. While there are outstanding questions (Dobs, Isik, Pantazis, \& Kanwisher, 2019; Young \& Burton, 2018), there is good comprehension of processes involved in accurate identity recognition. In contrast, there remain gaps in understanding the contribution of underlying perceptual mechanisms to accurate emotion recognition (Bombari et al., 2013; Meaux \& Vuilleumier, 2016) despite a degree of shared processing pathways in the early stages of facial identity and emotion recognition (Connolly, Young, \& Lewis, 2018). Some insight into underlying mechanisms of interpreting facial expressions can be gleaned from comparison studies of groups with and without impairment in face emotion recognition. One such group with a deficit in face emotion recognition is people with Parkinson's disease (Argaud, Vérin, Sauleau, \& Grandjean, 2018).

Parkinson's disease (PD) is primarily a disorder of later life. The major manifestation is progressive disturbance in motor function caused by dopaminergic cell loss in the substantia nigra, which projects to the striatum, with formation of Lewy bodies (Bradshaw \& Mattingley, 1995). During the course of this progressive disease damage extends from the nigrostriatal system to many other regions; and although the classic triad of tremor, rigidity and bradykinaesia remains the hallmark of the disease first described by Parkinson (1817), various forms of cognitive 
impairment become manifest, even in those who are newly diagnosed (Baggio et al., 2012). One of these cognitive deficits is impaired face perception and memory (Cousins, Hanley, Davies, Turnbull, \& Playfer, 2000), and in recognising emotional expressions (Argaud et al., 2018; Baggio et al., 2012; Narmé, Bonnet, Dubois, \& Chaby, 2011; Péron, Dondaine, Le Jeune, Grandjean, \& Vérin, 2012).

Whilst contradictory results pervade the literature regarding the extent of impairment in face emotion recognition in people with Parkinson's, Péron et al.'s systematic review of 43 published studies examining emotion processing in PD patients concluded that impairment is greater for negative emotions - anger, disgust, fear and sadness - than relatively positive emotions - happiness and surprise (Péron et al., 2012). This can be understood, they argued, pathophysiologically through disruption to normal functional involvement of dopaminergic pathways and the basal ganglia. More lately, this lab extended their review to 97 studies comparing facial emotion recognition in individuals with PD and controls (Argaud et al., 2018). They suggested that the origins of inconsistencies in the literature are at least partly explained by variations in methodology and they raised the question of whether the sensitivity of some tasks that have been used was sufficient to reveal impairments. Critically, however, this review concluded that there was strong evidence of an overall deficit in facial emotion recognition in PD, and that this deficit was greatest for the negative emotions.

Both individual facial features (i.e. eyes, nose and mouth) and the spatial relationship between these features are important in recognising faces (Carey \& Diamond, 1977; Rakover, 2002; Tanaka \& Simonyi, 2016). The literature presents evidence supporting holistic, configural processing of whole, upright faces occurring automatically, which enables almost instant overall recognition of a face at the 
expense of attention to face parts (Tanaka \& Farah, 1993; Thompson, 1980).

Originally the description of a face as providing 'configural information' was used by Carey and Diamond (1977) simply to refer to the layout or positioning of the features of a face. Later, these authors (Diamond \& Carey, 1986) described two types of configural information: "first order relational properties" (p.110) which referred to the basic layout of features that distinguishes a face from other stimuli, and is common to all faces (i.e., two eyes above a nose, which is above a mouth); and "second order relational properties" (p.110) which refer to the subtle differences in spacing and feature size and shape which allows a face to be distinguished from other faces. Subsequently, it has been proposed (Maurer, Le Grand, \& Mondloch, 2002) that there are three components that underpin configural processing. Similar to Diamond and Carey (1986), Maurer et al., (2002) distinguish between "sensitivity to first-order relations" (p.255): detection of face-like relations in stimuli; and "sensitivity to second-order relations" (p.255) referring to perceived variations in internal features and spacing. However they also claim that detection of face first-order relations is on the basis of holistic processing utilizing Gestalt principles of closure, and that it is this immediate processing response that undermines processing of individual features of a face that comes into view.

Upright face processing has great evolutionary importance and, in practice, an obligatory skill for most people (Cousins, 2013). This is the natural orientation, and many experimental studies have convincingly confirmed findings that when a face is presented upside-down, recognition is seriously disrupted (e.g., Rossion, 2008; Valentine, 1988; Yin, 1969). The Face Inversion Effect (FIE), as it is known, refers to the substantial decrease in recognition ability when a face is not presented in the upright orientation. Understanding this phenomenon has been the subject of much 
investigation, and whilst not fully understood, there is some consensus that the FIE has a perceptual basis underpinned by disruption to the normal ability to holistically process face relational information (Richler, Mack, Palmeri, \& Gauthier, 2011; Rossion, 2008).

Cousins et al. (2000) examined the possibility that face memory problems seen in people with PD are the result of impairment in configural processing using an adaptation of Mooney's Face Closure Test (Mooney, 1957). 40 black and white drawings of human faces which disclosed only the salient highlights or shadows were presented upright as target closure items or inverted as distractors. The test required participants to first determine whether the given stimulus was a face, then if so, to reveal guessing, participants were asked to identify age group (young, adult, old), and sex (male or female). Even though the stimuli provide only "a confused or incomplete configuration" (p.157), Mooney's faces are recognised immediately (Schwiedrzik, Melloni, \& Schurger, 2018) by neurotypical individuals. Cousins et al. however, found their PD group had significantly greater difficulty performing this task than a control group. Later, on the basis that some of the early perceptual processes required for the structural encoding faces are involved in both identity and emotion recognition, Narmé et al. (2011) suggested that this configural processing impairment may also explain deficits in face emotion recognition in PD.

Narmé et al. (2011) compared the performance of 12 non-dementing, fully medicated Parkinson's patients and 10 controls on an emotion recognition task. Ten Ekman faces representing four basic emotions: happiness, fear, disgust, anger, as well as a neutral expression (50 photographs) were presented both upright and inverted. Narmé et al.'s procedure was that images remained on screen until the participant selected one of the five emotion expression labels. They found that for 
upright faces their PD group were less accurate than their control group, and for inverted faces there was no difference in performance between the two groups. They also reported a significantly larger effect of inversion in the controls than in the PD group. Consistent with the view that the processing of inverted faces depends on featural processing, similar performance by the two groups was taken as support for their hypothesis. That is, featural processing ability was normal in the PD group, and an impairment in configural processing was responsible for the observed emotion recognition deficits.

Narmé et al. (2011) based their assertion on pooled data from four emotions. If there was a difference in reliance on configural processing according to emotion, then this may have been an inappropriate conclusion. Narmé et al. reported happiness judgements at ceiling (99\% accurate), and for upright faces, the PD group were significantly impaired compared to their controls only in classifying anger. This means they were unable to replicate previous findings of impairment in recognition of fear and disgust in their PD sample (c.f., Argaud et al. 2018; Péron et al., 2012). Support for Narmé et al.'s conclusion then, is relatively weak, indicating a need to replicate their study, including an examination of the impact of configural processing disruption on recognition of negative face expressions separately.

Alonso-Recio, Martín, Rubio, and Serrano (2014) used two types of task to examine the role of configural processing in facial emotion recognition in PD. They used a discrimination task - in which participants had to decide whether two faces displayed "same" or "different" emotions (anger, disgust, fear, sadness or happiness from FACES Database) - and a categorisation task, in which participants had to decide which emotion a face showed. The natural coloured face stimuli were displayed until a response was made or for up to 10 seconds. They found no 
difference between people with PD and controls in the discrimination task, but the performance of the Parkinson's group was significantly poorer than the control group on the categorisation task. Alonso-Recio et al. suggested that good performance on the discrimination task indicated that the use of configural processes in facial emotion recognition by patients with PD is not globally impaired. This claim rests on the assumption that both tasks required some form of configural processing. Such an interpretation can, however, be contested (e.g. De Haan \& Nelson, 1998). Instead, it may be the case that the discrimination task was more sensitive to a comparison of facial features while the categorisation task was more sensitive to configural processing. The main results of the study were derived from pooled face emotions data. The authors also provided information on correct categorisation of the five emotions separately. These differed according to target emotion. As with Narmé et al. (2011) means were highest for happy and lowest for anger. Altogether, AlonsoRecio et al.'s findings are not necessarily inconsistent with the view that poor emotion recognition in PD is caused by a configural processing deficit.

Argaud et al. (2018) discussed differences in recognition performance among emotions in PD. We suggest these differences can be attributed to the role of configural processing in achieving recognition of an emotional expression. That is, differences in findings between emotions may be a result of some emotions being more reliant upon configural processing (McKelvie, 1995; Prkachin, 2003). McKelvie (1995) examined the performance of undergraduate students on recognition of emotion expressions in upright and inverted faces. He found that their ability to recognise facial expressions was not completely lost with inversion, and that the negative effect of inversion varied across the six basic emotional expressions he used. McKelvie argued that the difference accorded to dependency of the specific 
emotion on configural information for identification. He found virtually no impact of inversion on happy expressions, in contrast to significant misidentification of sadness and anger expressions. Prkachin (2003) similarly found negative emotions to be more reliant on configural processing for recognition with a greater effect of inversion for anger and fear, than for disgust; sadness was intermediate. Critically, she concluded that a more intense search of both eye and mouth regions is required for identification of anger, disgust, fear and sadness expressions than other expressions. That is, these studies suggest that the demands of configural processing vary across emotion expression in the face, and this may underpin the selective deficits in emotion expression recognition seen in PD.

Face inversion is generally defined as a rotation of a face through $180^{\circ}$ so that it is upside down. There is no change to either features or configuration. This has raised various explanations of how this manipulation prevents instant recognition. Relevant to this exploration, Diamond and Carey (1986) argued that the detrimental effects of inversion are confined to configural processing. Much evidence is consistent with disruption to configural processing (see Rakover, 2013 for review). However, there is also accumulating evidence that inversion does not spare featural, part-based processing of faces, as has generally been assumed. Some of the most convincing evidence that facial features are vulnerable to the effects of inversion was provided by Psalta, Young, Thompson, and Andrews (2014). Their study examined the effects of inversion on the processing of grotesque Thatcherised faces. Psalta et al. pointed out that the inability to perceive grotesque expressions in inverted Thatcherised faces has generally been attributed to the disruption of $2^{\text {nd }}$ order relations by inversion. However, in their study, the ability to detect a grotesque feature was disrupted by inversion even when only the mouth region of a 
Thatcherised face was presented. Psalta et al. suggested that the Thatcher illusion is best explained in terms of inversion reducing sensitivity to the perception of facial features. But if inversion affects featural processing, then it is perhaps surprising that Narmé et al.'s (2011) PD sample showed such a small effect of facial inversion. If individuals with PD rely on featural processing, and if featural processing is disrupted by inversion, then it would be expected that individuals with Parkinson's disease confirm a substantial inversion effect when attempting to recognise facial expressions. Moreover, there are also doubts as to whether inversion abolishes configural processing. For example, Rivest, Moscovitch, and Black (2009) argued that is possible to access some configural information from a face even when it is inverted. So, if controls can access some configural information from an inverted face, then they would be expected to out-perform individuals who suffer from a configural processing deficit. Inversion, therefore, may not provide a particularly sensitive way of investigating whether individuals with Parkinson's disease have a configural processing impairment.

Consequently, our study used an additional task to investigate this issue further; individuals with Parkinson's disease were asked to identify emotional expressions from facial composites. In the composite paradigm (Young, Hellawell, \& Hay, 1987), faces of individuals are divided to form a top and a bottom half. The halves from two different individuals can be aligned together. Under these circumstances, the identity of the person in the top or bottom half of the composite is recognised more slowly and less accurately than when the two segments are nonaligned - that is, the top half and bottom half of a face do not line up, and the mouth could be under an ear. It appears that configural processing fuses the two halves of aligned composites into a novel Gestalt that interferes with recognition from the top 
or bottom half of the face. The composite paradigm demonstrates the challenge of attending to just a part of a face in the context of a whole face, thereby revealing the significance of configural processing in face recognition.

Although originally developed to examine identity recognition, there have since been various iterations of the face composite effect methodology. Calder, Keane, Young, and Dean (2000) presented composites in which the top and bottom halves showed the same person's face displaying two different emotions. The task was to identify the emotion being displayed in either the top or bottom half of the composite. The composites were aligned on some trials and non-aligned on others. Calder et al. (2000) found a composite effect in both facial emotion recognition and facial identity tasks in their sample of 12 young participants; accuracy was lower, and reaction times were higher in the aligned condition compared to the nonaligned condition. Poorer performance in the aligned condition was attributed to a difficulty in ignoring 'irrelevant' information about expression from the facial composite. If individuals with Parkinson's disease are impaired at configural processing then it would follow that, when judging the emotion associated with the top half of a composite, they would be less likely than controls to suffer interference from the bottom half when it is displaying a different emotion (and vice versa). We would therefore expect to observe relatively good performance in the PD sample when processing aligned composites leading to a reduced or abolished facial composite effect.

In summary, this study sought to explain why individuals with Parkinson's disease are impaired at judging emotional expressions from faces. To examine the role of configural processing in emotion recognition we used anger, disgust, fear, and sadness face stimuli both to reflect the PD literature (Péron et al., 2012; Argaud 
et al., 2018), and because these emotion expressions may be more dependent upon configural processing (Prkachin, 2003). The study investigated whether a PD group would show a significantly smaller facial composite effect than controls. It also sought to replicate previous findings that expression decisions in individuals with Parkinson's disease are relatively insensitive to the effects of facial inversion. Specifically, we hypothesised:

1. Controls will perform significantly better in upright whole face emotion recognition than people with Parkinson's.

2. There will be no Face Inversion Effect (FIE) in the Parkinson's group.

3. Controls will have significantly better face emotion recognition for non-aligned composite faces than for aligned composite faces.

4. There will be no composite effect for the Parkinson's group.

\section{Methods}

Ethical approval for the study protocol was granted by a National Health Service Health Research Authority National Research Ethics Committee.

\section{Design}

We used a prospective ex post facto study with an interparticipant independent variable: Group (PD and Control Group) and two intraparticipant independent variables: compositive (aligned and non-aligned) and composite faces (upright and upside-down). Dependent variables were accuracy and the recognition of emotion expression. 


\section{Participants}

A purposeful sampling technique (Bowling, 2014) of people with confirmed PD with a range of age, disability, and disease duration was employed. Participants were excluded from the experimental sample if they also had a diagnosis of concomitant dementia or any other neurological condition in conjunction with PD. 18 people with idiopathic PD and 18 controls matched for age and current verbal intelligence at time of testing consented to take part. No payment was made to any participant. All participants were Caucasian and lived in North-West England. Two participants (one $\mathrm{PD}$, one control) were not recruited following screening for compromised cognition, and one PD participant withdrew during testing. The final sample therefore contained 16 people with PD and 17 controls. Sample size calculations are sensitive to error and complicated when drawing upon incomplete information in the literature (Noordzij et al., 2010). This sample size was calculated to be sufficient at an alpha of 0.05 and a power of .80 , to test for a difference between the PD group and a matched control group based on previous published research output, particularly that of Narmé et al. (2011), whose research we were replicating and extending. Narmé et al. (2011) found relevant significant differences in emotion expression recognition between groups of twelve people with PD and ten controls.

Table 1 contains details of the demographics of the sample.

Insert Table 1 here

The PD group were all receiving dopaminergic medication at the time of testing (14/16 were taking levodopa preparations, 11/14 were also taking dopamine 
boosting drugs; 2/16 were taking dopamine agonists only); none were distracted by tremor or drug-induced dyskinaesias during the testing session.

The control group comprised people without neurological illness who were similar in age and background to the case sample. Family caregivers were recruited where possible with additional purposive recruitment from healthy volunteers known to the researchers.

There was no statistical difference between the PD patients and the controls in terms of age or current verbal intelligence as assessed by the Mill Hill Vocabulary Test (Raven, 1943). The Mill Hill test investigates knowledge of up to 17 target words that progress in difficulty. Participants are asked to select the best synonym from a choice of six words that accompany a given target word. Most participants were willing and able to guess from alternatives if they did not immediately know the target word, and they were not disabused of any errors. After three successive errors, the test was terminated.

No attempt was made to match the two groups using a test of nonverbal intelligence. As previously reported (Cousins et al., 2000), tests of non-verbal intelligence disadvantage people with Parkinson's because of these tests rely on visual closure. In an investigation such as this, there would therefore be a risk of contaminating dependent variables if a non-verbal test were used to match participants.

No attempt was made to match the two groups for depression even though depression is frequently a co-morbid condition for Parkinson's patients (Schrag et al., 2007). PD samples are generally more depressed than healthy control samples, but severity is usually classified as "mild" (Mayeux, 1981). Lees (1990) cautions that some symptoms of PD provide opportunity for misdiagnosis of depression, that in 
most patients with depression, it is relatively mild, and psychotic depression occurs in less than $10 \%$ of cases. In their review of use of depression rating scales used in PD, Schrag et al. (2007) discuss problems with using depression rating scales in PD, and note that the typical inclusion of somatic items can unduly inflate scores, and inappropriately "influence the results of treatment trials of depression in PD" (p1087). We proceeded by ascertaining full medication intake of all participants, with a view to excluding any participant with a history of clinical depression. We included two participants with PD and one Control participant who were currently taking a low dose of antidepressants, and one Control participant who was taking anti-anxiety medication.

\section{Materials}

The faces of 10 Caucasian people ( 5 male) were selected from the Radboud Faces Database (Langner et al., 2010). Four natural colour photographs of each face were used, making a total of 40 photographs. Each photograph displayed one of four basic emotions - anger, disgust, fear, sadness - described by Ekman and Friesen (1975). That is, for 'anger', the eyes were bulging and the lips were pursed; for 'disgust' the nose bridge was wrinkled, the cheeks were raised and the upper lip was raised; the 'fear' expression was distinguished by wide open eyes, raised brows and an open mouth; and in the 'sadness' expression the eyes were framed by raised inner brows and the corners of the mouth were lowered.

For the composite stimuli, the 40 photographs were cut horizontally across the middle of the face, directly under the nose so that the eyes and the mouth were in separate parts: the eyes and the mouth are most important for expression recognition (Tanaka \& Simonyi, 2016). Composites were made from the same 
models for 12 different eyes/mouth emotion combinations: anger/disgust, anger/fear, anger/sadness; disgust/anger, disgust/fear, disgust/sadness; fear/anger, fear/disgust, fear/sadness; sadness/anger, sadness/disgust, sadness/fear. The aligned composites were edited where necessary - using Adobe Photoshop - to occlude differences in shade, and ensure there were no gaps, or sharp edges at the jawline or on the nose. Non-aligned composites were made following the principle of moving the top half to the left or to the right until the model's jawline was directly under the middle or the nose, and with no gap between the two parts. Examples of the face stimuli are presented in Figures 1, 2, and 3.

Insert Figures 1, 2, 3 about here

All stimuli were presented on a 17" high-resolution monitor, where each image was presented in a portrait orientation and occupied $21 \mathrm{~cm} \times 14.5 \mathrm{~cm}$ (non-aligned $21 \mathrm{~cm} \times 17.25 \mathrm{~cm}$ ) against a plain white background. Viewing distance was at each participant's preference. All participants used their best-corrected vision for inspecting the stimuli.

\section{Procedure}

Three experimental tasks were created to test the hypotheses.

1. Full face emotion expression recognition: upright and inverted stimuli. There were 80 different trials. The 40 photographs -10 for each emotion - were all presented once upright and once inverted. The order of presentation was randomly assorted to prevent anticipation of next emotion response. All 
participants were presented with the stimuli in the same order. The task was to correctly identify the facial emotion (anger, disgust, fear, sadness).

2. Non-aligned composite face emotion expression recognition.

3. Aligned composite face emotion expression recognition

For both composite face tasks there were 96 different trials: 12 different eyemouth combinations of the four emotions, each upright and inverted. Each emotion appeared top 24 times, and bottom 24 times, and the emotion to be identified was top 48 times and bottom 48 times.

People with a diagnosis of PD have bradykinaesia. As such, collection of reaction time responses is an inappropriate methodology for comparing performance of a PD with healthy controls (Bloxham, Dick, \& Moore, 1987; Wearden et al., 2008). Our validity assessment of the testing protocol indicated that people with PD need more time to respond, even verbally, than given in similar tests of face emotion expression recognition in the literature. Equally, allowing an untimed free response could permit sufficient featural processing to mask any impairment in configural processing. We managed this situation by providing a clear response time between stimuli presentations. That is, in each of the three tasks, participants were presented with a full face or composite face for 3000 milliseconds and this was followed by an inter-trial interval of 3000 milliseconds. Together, this provided a 6000 milliseconds time window for participants to make one of the four given two-syllable verbal responses according to their perception of the face or composite part (i.e. "anger", "disgust", "fearful", "sadness"). In the full-face test, the screen was blank during the 3000 milliseconds inter-trial interval, whereas in the two composite tests, either 'TOP' or 'BOTTOM' was displayed in the centre of the screen to indicate which half of the 
composite they should state "anger", "disgust", "fearful", or "sadness", according to their perception, in the next trial.

As the sample size was not large enough to counterbalance three tests with different cognitive load, we took the view that a better approach to manage the between group comparisons was to use the same test order for all participants. That is, the full-face task was conducted first, then the non-aligned composites, and finally aligned composites. The sequence of the photographs in each of the three tests was randomised, then loosely managed to prevent multiple trials requiring the same response which could hide perseveration to which PD patients are prone (e.g., Lees \& Smith, 1983).

Testing took place in a single session in the participant's home. The PD group were not asked to withhold their normal medication, and the experimenter ensured participants remained 'on' before starting each task. The three tasks were run as three timed presentations, as above, without pause. Rest periods between the three tasks were as long as required. Before each test, participants were given a practice set of 12 stimuli, untimed and with feedback. When participants confirmed they understood the procedure, formal data collection began. No feedback was given of performance in any of the tests.

Verbal responses were recorded on prepared score sheets. Data were then entered into SPSS v25.0 database for statistical analyses. Where there were violations of assumptions of normality, non-parametric tests were used. As the analyses was restricted to a small number of planned comparisons, we followed advice (Armstrong, 2014; Djulbegovic et al., 2015) that Bonferroni corrections to alpha levels would be an overly conservative procedure. Hence, we worked with conventional level of significance setting alpha at .05. 


\section{Results}

Full-face emotion expression recognition

Insert Table 2 here

Table 2 provides mean scores for the four emotions, according to group and orientation. Shapiro-Wilk tests indicated violations to assumptions of normality in some of the whole face data: for Anger upright and Sad upright for both groups, and Disgust inverted for the PD group. In view of this, non-parametric Mann-Whitney analyses (with one-tailed Exact significance levels) were used for whole face group comparisons. For upright faces the PD group exhibited impairment in recognition of Anger $(U=59.5 ; p=.002)$, Disgust $(U=69.0 ; p=.007)$, and Fear $(U=81.0 ; p=$ .023). The difference between the groups for Sadness was not statistically significant. For inverted faces, controls were significantly more accurate than people with Parkinson's for Disgust $(U=68.0 ; p=.007)$ and Sadness $(U=73.5 ; p=.011)$.

Paired Wilcoxon signed-ranks analyses (with 1-tailed Exact significance levels) of recognition of upright and inverted faces according to emotion indicated that both groups were significantly better in the upright orientation (see Table 2).

A 2 (group) $\times 4$ (emotion type) $\times 2$ (orientation) repeated measures general linear model analysis confirmed small between group differences $\left(F_{(1,31)}=7.78 ; p<\right.$ $\left..009, \eta_{p}^{2}=.20\right)$; medium emotion type differences $\left(F_{(1,31)}=37.81 ; p<.009, \eta_{p}^{2}=\right.$ $.55)$; and large orientation differences $\left(F_{(1,31)}=80.80 ; p<.001, \eta_{p}^{2}=.72\right)$ across the whole sample. There was no group $\mathrm{x}$ emotion type interaction, no group $\mathrm{x}$ orientation interaction, and no group $\mathrm{x}$ emotion type $\mathrm{x}$ orientation interaction. 


\section{The Composite Paradigm}

Data from two tests examining face emotion expression recognition in composites are summarised in Table 3 . The data did not violate assumptions of normality.

Insert Table 3 here

In the conventional upright condition, when considering the four negative emotion expressions together, the control group was significantly more accurate when composite face stimuli were not aligned than when aligned $\left(t_{(1,16)}=-2.46 ; p=\right.$ .026). For the PD group this composite effect was absent. Emotion specific analyses found no composite effect for anger, disgust or fearful for the PD group, although for sadness recognition was better for non-aligned composites $\left(t_{(1,15)}=-2.37 ; p=.03\right)$. For the control group, a significant composite effect was found for disgust and fearful, but not anger or sadness.

Examination of the inverted composite stimuli confirmed that there were no composite effects at all. Across every emotion and condition there was a decrement in performance in both groups when compared to performance when composites were upright.

\section{Discussion}

Our findings of a primary impairment in people with PD in recognising anger, disgust and fearful are largely in line with other studies which have analysed negative face emotion recognition separately, rather than as a single phenomenon (Arguad et al., 2018). To examine whether a configural processing deficit was involved in these impairments, we employed two established tests of configural processing: inversion 
and half-face composites. We reasoned that if impairment in face emotion recognition was associated with a configural processing deficit, then people with PD should more readily perceive the target emotion in top or bottom of an aligned composite 'face'. That is, a PD group should be much less inconvenienced than controls when top and bottom halves of a composite are showing different emotions. Consistent with this prediction, our results revealed a significant overall composite effect for controls but not for the PD group: the controls outperformed the PD group with nonaligned but not with aligned composites. This outcome provides new evidence that the impairment in recognizing emotional expressions from faces in PD is associated with impaired configural processing in the context of preserved featural processing.

We hypothesised that for inverted faces, there would no longer be an advantage for the control group, and a face inversion effect would be found in the control group, but not in the PD group. However, significant effects of face inversion were observed in both groups. Inversion effects were present with standard presentation of faces (Table 2), and with aligned and nonaligned facial composites (Table 3). Given that inversion makes it difficult to access configural information from a face, it might have been expected that emotion recognition would rely on featural processing. If so, it would have followed that the PD group would have shown a relatively small inversion effect and would have performed significantly worse than the controls with upright but not with inverted faces. These were precisely the results obtained by Narmé et al. (2011). However, our PD group performed significantly worse than controls on both upright and inverted faces and the effect of inversion was approximately equal in the PD sample and the controls. 
Why might our results have differed from those reported by Narmé et al. (2011)? It is important to point out that their PD sample did perform significantly worse with inverted than with upright faces (see Table 2, Page 3298) even if this effect was significantly smaller than that shown by their control group. One obvious methodological difference is that whereas our study only examined negative emotions, Narmé et al. included happy faces which their PD sample recognised correctly as being happy faces. Moreover, performance on only one of the four emotions that they examined was clearly impaired in Narmé et al.'s PD group. This means that our study produced a more powerful between-group effect of emotion recognition that may have made it easier for us to detect a strong effect of inversion in our PD sample. It is also interesting to note that Narmé et al.'s PD group performed particularly well relative to controls with inverted happy and inverted fear faces and particularly badly with upright anger faces. Precisely why this was the case is unclear, other than that it is an outcome of differing demands on configural processing in recognising positive (happy) and negative (anger) stimuli (McKelvie, 1995; Prkachin, 2003).

The fact that people with PD in our study showed an effect of inversion but not of facial composites suggests that these two tasks are sensitive to different experimental variables. This outcome has important implications for our understanding of the types of processing that these two tasks require. How is the difference between the results with inverted faces and facial composites best understood?

One possible explanation is that these two tasks disrupt different aspects of configural processing. Richler and Gauthier (2014) have pointed out that the terms 'configural' processing and 'holistic' processing are often used synonymously, and 
that both have been used as a blanket term for the perceptual encoding of faces. It has been suggested, however, (e.g. Maurer et al., 2002; Mestry, Menneer, Wenger, \& Donnelly, 2012; Richler \& Gauthier, 2014) that the perception of faces may require more than one type of 'configural' processing. According to Mestry et al., one should distinguish the ability to perceive a face as a whole - 'holistic processing' - from the use of second-order relations between the locations of the internal features of a face to recognize facial expression or identity. Holistic processing is seen as being responsible for the improved ability to recognize a familiar facial feature when that feature appears in the context of a whole face than when it appears in isolation (Tanaka \& Simonyi, 2016). Configural processing requires sensitivity to second order relations between the internal features of a face that are seen as being responsible for identifying a familiar person (Moscovitch, Winocur, \& Behrmann, 1997). Importantly, Maurer et al. (2002) argued that performance with facial composites is sensitive to the effects of holistic rather than configural processing. The absence of a facial composite effect in our PD group therefore provides strong evidence that in these terms they have a problem in processing faces holistically.

If it is assumed that a normal inversion effect means that configural processing is unimpaired, then one explanation of the large inversion effect in our PD group is that they suffer from a holistic processing deficit but the three types of configural processing deficit were not equally negatively affected. That raised the question of whether there is any evidence to support the claim that inversion disrupts second order relational processing (Maurer et al., 2002). One source of corroboration can be seen in a study by Searcy and Bartlett (1996). They investigated facial emotion recognition in a series of timed comparison tasks and found that inversion reduced the speed of same / different responses to faces with 
altered spatial relations significantly more than faces with distorted features (eyes and mouths). Their results indicated that inversion significantly disrupts the ability to extract second order relational information from a face. Also, holistic processing would appear to operate at a lower level than the detection of second order relations in a face, and as such, any mechanism that disrupts holistic processing would also impair configural processing. As Maurer et al. (2002) pointed out, no data exist to refute this possibility.

There is, however, a simpler alternative explanation: the significant inversion effect that we observed in our PD group is consistent with them suffering from both a holistic and a second order relational processing deficit. Inversion may simply provide a less sensitive way of selectively disrupting configural processing than the use of face composites. As we pointed out in the Introduction, it is possible that some configural information can still be accessed from inverted faces (e.g. Rivest et al., 2009). If so, then individuals with PD would perform worse than controls if controls are able to access configural information from inverted faces but they cannot. Individuals with PD would also perform significantly worse on inverted faces than upright faces if inversion impairs featural processing (Psalta et al., 2014) on which they are particularly reliant in order to make accurate decisions about facial emotions.

People with Parkinson's disease were impaired in the recognition of the anger, disgust, and fearful facial emotions. The finding that people with Parkinson's showed no difference in their ability to identify emotional expressions when half-face composites were presented aligned or non-aligned means that their problems in recognising facial expressions can be more readily explained in terms of a configural processing deficit. However, despite showing no composite effect, our PD sample 
showed a strong inversion effect when attempting to recognise facial expressions. Our results therefore suggest that inversion and the use of composites disrupt face processing in different ways. One explanation of the finding that people with PD showed impaired emotional recognition even when faces were inverted is that inversion disrupts configural processing (detection of second order relations) whereas composites disrupt holistic processing (the ability to see a face as a whole). It is therefore possible that even though our PD sample had a holistic processing deficit they did not have a configural processing deficit. There is, however, a plausible alternative explanation. If inversion can significantly disrupt featural processing as well as configural processing (Psalta et al., 2014), and if some configural information is available to controls even when faces are inverted (Rivest et al., 2009), then an inversion effect would be predicted even in individuals with impaired configural processing. It therefore remains likely that individuals with PD have both a configural and a holistic processing impairment. If so, the significant inversion effect on facial emotion recognition that we observed in individuals with Parkinson's is consistent with our original hypothesis that they rely disproportionately on featural processing when perceiving faces.

The PD face emotion expression recognition literature has been challenged by many differences in sample selection and methodology. It could be argued that we have added another. However, we defend this, pointing out that we have used a validated set of natural faces as stimuli in a robust paradigm. We extended stimuli exposure time to compensate for Parkinson's bradykinaesia, but not at the expense of reducing validity. Moreover, we did not want to succumb to the same criticism reported in the literature of low levels of difficulty, lack of task sensitivity, and ceiling effects which have probably concealed deficits between Parkinson's and controls in 
at least six studies which did not find difference on emotion recognition (Argaud et al., 2018). There are also many studies where a significant difference between Parkinson's and controls relies on reaction time data. A strength of our study is that we process validated our procedure to ensure sufficient difficulty as to avoid ceiling effects, and we provided enough time to avoid guessing and potentials for performing at chance level. Feedback from our small feasibility study led to the inclusion of the empty slide between stimuli for responding without compromising the underpinning premise that emotion expression recognition takes place quickly in the real world, when efficient and effective. In the test situation, it was clear that the experiment was challenging, but doable.

There is also a view that neuropsychological explanations using PD samples may not be reliable, as impairment could be due to general cognitive deficits in the Parkinson's population being studied. Nevertheless, although PD is a progressive disease, specific cognitive deficits can be seen in a non-demented PD sample even at very early stages of the disease (e.g., Hietanen \& Teravainen, 1986; Lees \& Smith, 1983), including face recognition (Cousins et al., 2000; Dewick, Hanley, Davies, Playfer, \& Turnbull, 1991). For both these reasons, we report differences in Hoehn \& Yahr stage in our sample, but examining differences in emotion expression recognition according to this disease progression classification is beyond the scope of this research. It is evident that there is a spectrum of cognitive impairment in the Parkinson's population, ranging from no observable deficit to concomitant dementia (Litvan et al., 2012). Litvan et al. attempted to develop a procedure to diagnose Parkinson's disease mild cognitive impairment (PD-MCI) which is purported to precede Parkinson's disease dementia. However, their guidelines have been criticised in longitudinal studies using their diagnostic criteria for PD-MCI for being 
poor at identifying the high risk-dementia profile as intended (Biundo, Weis, \& Antonini, 2016). A challenge is that the heterogeneous nature of cognitive decline in Parkinson's does not allow clear understanding of 'pre-dementia'. We accept that controlling for potentials for pre-dementia is not straightforward: our recruitment method was to first screen out any potential participants with clear cognitive decline / PDD, then to mitigate for potential PD-MCI by matching our experimental PD sample with a healthy control sample on a measure of current intelligence (i.e., Mill Hill test). We argue that this is suitable and sufficient at this time to ensure any comparison of cognitive performance is valid.

We provided a substantial test of configural processing using two paradigms for four negative emotions. The decision to limit the stimuli to four negative facial emotions, rather than including positive and neutral facial emotions could be construed as a limitation of the study. The rationale was based on the previous literature, which indicated that different emotions have different structural correlates (Baggio et al., 2012), and, where deficits have been found these have been limited to the negative emotions (Argaud et al., 2018; Péron et al., 2012; Prkachin, 2003). The different demand on cognitive resources of the three tests used in this paradigm suggested that counterbalancing the tests across participants was inappropriate, despite this being a more robust experimental procedure.

\section{References}

Alonso-Recio, L., Martín, P., Rubio, S., \& Serrano, J. M. (2014). Discrimination and categorization of emotional facial expressions and faces in Parkinson's 
disease. Journal of Neuropsychology, 8, 269-288.

https://doi.org/10.1111/jnp.12029

Argaud, S., Véron, M., Sauleau, P., \& Grandjean, D. (2018). Facial Emotion

Recognition in Parkinson's disease: a review and new hypothesis. Movement Disorders, 33, 554-567. https://doi.org/10.1002/mds.27305

Armstrong, R. A. (2014). When to use the Bonferroni correction. Ophthalmic Physiological Optics, 34, 502-508. https://doi.org/10.1111/opo.12131

Baggio, H. C., Segura, B., Ibarretxe-Bilbai, N., Valldeoriola, F., Marti, M. J., Compta, Y., .... \& Junque, C. (2012). Structural correlates of facial emotion recognition deficits in Parkinson's disease patients. Neuropsychologia, 50, 2121-2128. https://doi.org/10.1016/j.neurpsychologia.2012.05.020

Biundo, R., Weis, L., \& Antonini, A. (2016). Cognitive decline in Parkinson's disease: the complex picture. NPJ Parkinson's Disease, 2, 16018. https://doi.org/10.1038/npjparkd.2016.18

Bloxham, C. A., Dick, D. J., \& Moore, M. (1987). Reaction times and attention in Parkinson's disease. Journal of Neurology, Neurosurgery, and Psychiatry, 50, 1178-1183. https://doi.org/10.1136/jnnp.40.9.1178

Bombari, D., Schmid, P.C., Schmid Mast, M., Birri, S., Mast, F. W., \& Lobmaier, J. S. (2013). Emotion Recognition: the role of featural and configural face information. The Quarterly Journal of Experimental Psychology, 66, 24262442. https://doi.org/10.1080/17470218.2013.789065

Bowling, A. (2014). Research Methods in Health: Investigating Health and Health services (2nd $E d$.$) . Buckingham: Open University Press.$ 
Bradshaw, J. L., \& Mattingley, J. B. (1995). Clinical Neuropsychology: Behavioural and Brain Science. Sydney: Academic Press. https://doi.org/10.1016/C2009$\underline{0-02280-6}$

Calder, A. J., Keane, J., Young, A. W., \& Dean, M. (2000). Configural information in facial expression perception. Journal of Experimental Psychology: Human Perception and Performance, 26, 527-551. https://doi.org/10.1037/0096$\underline{1523.26 .2 .527}$

Carey, S., \& Diamond, R. (1977). From piecemeal to configurational representation of faces. Science, 195, 312-314. https://doi.org.10.1126/science.831281

Cousins, R. (2013). Prosopagnosia after stroke: potentials for impairment and treatment. Topics in Stroke Rehabilitation, 20, 471-477.

Cousins, R., Hanley, J. R., Davies, A. D. M., Turnbull, C. J., \& Playfer, J. R. (2000). Understanding memory for faces in Parkinson's disease: the role of configural processing. Neuropsychologia, 38, 837-847. https://doi.org/10.1016/S0028$\underline{3932(99) 00133-5}$

De Haan, M., \& Nelson, C. A. (1998). Discrimination and categorisation of facial expressions of emotion during infancy. In A. Slater (Ed.) Perceptual Development: Visual, Auditory, and Speech Perception in Infancy. Hove UK: Psychology Press Ltd.

Derntl, B., Seidel, E-M., Kainz, E., \& Carbon, C-C. (2009). Recognition of emotional expressions is affected by inversion and presentation time. Perception, 38, 1849-1862. https://doi.org/10.1068/p6448

Dewick, H. C., Hanley, J. R., Davies, A. D. M., Playfer, J. R., \& Turnbull, C. J. (1991). Perception and memory for faces in Parkinson's disease. Neuropsychologia, 29, 785-802. https://doi.org/10.1016/0028-3932(91)90072-G 
Diamond, R., \& Carey, S. (1986). Why faces are and are not special: an effect of expertise. Journal of Experimental Psychology: General, 115, 107-117. https://doi.org/10.1037/0096-3445.115.2.107

Dobs, K., Isik, L., Pantazis, D., \& Kanwisher, N. (2019). How face perception unfolds over time. Nature Communications, 10, Article number 1258. https://doi.org/10.1038/s41467-019-09239-1

Djulbegovic, M., Becjstead, J., Elqayam, S., Relijic, T., Kumar, A., Paidas, C., \& Djulbegovic, B. (2015). Thinking styles and regret in physicians. PLOS ONE, 10, 1-13. https://doi.org/10.1371/journal.pone. 0134038

Ekman, P. and Friesen, W. V. (1975). Unmasking the face: A guide to recognizing emotions from facial clues. Oxford, England: Prentice-Hall.

Hietanen, M., \& Teravainen, H. (1986). Cognitive performance in early Parkinson's disease. Acta Neurologica Scandinavica, 73, 151-195. Hyyps://doi.org/

Langner, O., Dotsch, R., Bijlstra, G., Wigboldus, D. H. J., Hawk, S. T., \& van Knippenberg, A. (2010). Presentation and validation of the Radboud Faces Database. Cognition \& Emotion, 24, 1377-1388. https://doi.org/10.1080/02699930903485076

Lees, A. J. (1990). The behavioural neurology of Parkinson's disease. In G. M. Stern (Ed.), Parkinson's Disease (pp. 389-413). London: Chapman and Hall Ltd.

Lees, A. J., \& Smith, E. (1983). Cognitive deficits in the early stages of Parkinson's disease. Brain, 106, 257-270. https://doi.org/10.1093/brain/106.2.257

Litvan, I., Goldman, J. G., Tröster, A. I., Schmand, B. A., Weintraub, D., Petersen, R. C., ... \& Emre, M. (2012). Diagnostic criteria for mild cognitive impairment in Parkinson's Disease: Movement Disorder Society Task Force guidelines. Movement Disorders, 27, 349-356. https://doi.org/10.1002/mds.24893 
McKelvie, S. J. (1995). Emotional expression in upside-down faces: Evidence for configurational and componential processing. British Journal of Social Psychology, 34(3), 325-334. https://doi.org/10.1111/j.2044-

\subsection{5.tb01067.x}

Maurer, D., Le Grand, R., \& Mondloch, C. J. (2002). The many faces of configural processing. Trends in Cognitive Sciences, 6, 255-260. https://doi.org/10.1016/S1364-6613(02)01903-4

Mayeux, R., Stern, Y. Rosen, J., \& Leventhal, J. (1981). Depression, intellectual impairment and Parkinson's disease. Neurology, 31, 645-650. https://doi.org/10.1212/WNL.31.6.645

Meaux, E., \& Vuilleumier, P. (2016). Facing mixed emotions: analytic and holistic perception of facial emotion expressions engages separate brain networks. Neurolmage, 141, 154-173. https://doi.org/10.1016/j.neuroimage.2016.07.004

Mestry, N., Menneer, T., Wenger, M. J., \& Donnelly, N. (2012). Identifying sources of configurality in three face processing tasks. Frontiers in Psychology, 3, 456. https://doi.org/10.3389/psyg.2012.00456

Mooney, C. M. (1957). Age in the development of closure ability in children. Canadian Journal of Psychology, 11, 219-226. https://doi.org/10.1037/h0083717

Moscovitch, M. Winocur, G., \& Behrmann, M. (1997). What is special about face recognition? Nineteen experiments on a person with visual object agnosia and dyslexia but normal face recognition. Journal of Cognitive Neuroscience, 9, 555-604. https://doi.org/10.1162/jocn.1997.9.5.555

Narmé, P., Bonnet, A-M, Dubois, B., \& Chaby, L. (2011). Understanding facial emotion perception in Parkinson's disease: the role of configural processing. 
Neuropsychologia, 49, 3295-3302.

https://doi.org/10.1016/j.neuropsychologia.2011.08.002

Noordzij, M., Tripepi, G., Dekker, F. W., Zoccali, C., Tanck, M. W., \& Jager, K. J.

(2010). Sample size calculations: basic principles and common pitfalls.

Nephrology Dialysis Transplantation, 25, 1388-1393.

https://doi.org/10.1093/ndt/gfp732

Parkinson, J. (1817). An Essay on the Shaking Palsy. London: Sherwood, Neely \& Jones.

Péron, J., Dondaine, T., Le Jeune, F., Grandjean, D., \& Vérin, M. (2012). Emotional processing in Parkinson's disease: a systematic review. https://doi.org/10.1002/mds.24025

Prkachin, G. C. (2003). The effects of orientation on detection and identification of facial expressions of emotion. British Journal of Psychology, 94(1), 45-62. https://doi.org/10.1348/000712603762842093

Psalta, L., Young, A. W., Thompson, P., \& Andrews, T. J. (2014). Orientationsensitivity to facial features explains the Thatcher illusion. Journal of Vision, 14, 1-10. https://doi.org/10.1177/0956797613501521

Rakover, S. S. (2002). Featural vs configural information in faces: a conceptual and empirical analysis. British Journal of Psychology, 93, 1-30. https://doi.org/10.1348/000712602162427

Rakover, S.S. (2013). Explaining the face-inversion effect: the face-scheme incompatibility (FSI) model. Psychonomic Bulletin \& Review, 20, 665-692. https://doi.org/10.3758/s13423-013-0388-1

Raven, J. C. (1943). The Mill Hill Vocabulary Scale. London: H. K. Lewis. 
Richler, J. J., \& Gauthier, I. (2014). A meta-analysis and review of holistic face processing. Psychological Bulletin, 140, 1281-1302.

\section{https://doi.org/10.1037/a0037004}

Richler, J. J., Mack, M. L. Palmeri, T. J., \& Gauthier, I. (2011). Inverted faces are (eventually) processed holistically. Vision Research, 51, 333-342. https://doi.org/10.1016/j.visres.2010.11.014

Richler, J. J., Palmeri, T. J., \& Gauthier, I. (2012). Meanings, mechanisms, and measures of holistic processing. Frontiers in Psychology, 3, 553. https://doi.org/10.3389/fpsyg.2012.00553

Rivest, J., Moscovitch, M., \& Black, S. (2009). A comparative case study of face recognition: The contribution of configural and part-based recognition systems, and their interaction. Neuropsychologia, 47, 2798-2811. https://doi.org/10.1016/j.neuropsychologia.2009.06.004

Rossion, B. (2008). Picture-plane inversion leads to qualitative changes of face perception. Acta Psychologica, 128, 274-289.

https://doi.org.10.1016/j.actpsy.2008.02.003

Schrag, A., Barone, P., Brown, R. G., Leentjens, A. F. G., McDonald, W. M., Startstein, S., .... \& Goetz, C. G. (2007). Depression rating scales in Parkinson's disease: critique and recommendations. Movement Disorders, 22, 1077-1092. https://doi.org/10.1002/mds.21333

Searcy, J. H., \& Bartlett, J. C. (1996). Inversion and processing of component and spatial-relational information in faces. Journal of Experimental Psychology: Human Perception and Performance, 22, 904-915. https://doi.org/10.1037/0096-1523.22.4.904 
Tanaka, J. W., \& Farah, M. J. (1993). Parts and wholes in face recognition. The Quarterly Journal of Experimental Psychology Section A: Human Experimental Psychology, 46, 225-245.

https://doi.org.10.1080.14640749308401045

Tanaka, J.W., \& Simonyi, D. (2016). The "parts and whole" of face recognition: a review of the literature. The Quarterly Journal of Experimental Psychology, 69, 1876-1889. https://doi.org/10.1080/17471218.2016.1146780

Wearden, J. H., Smith-Spark, J. H., Cousins, R., Edelstyn, N. M. J., Cody, F. W. J., \& O’Boyle, D. J. (2008). Stimulus timing by people with Parkinson's disease. Brain and Cognition, 67, 264-279. https://doi.org/10/1016/j.bandc.2008.01.010

Yin, R. K. (1969). Looking at upside-down faces. Journal of Experimental Psychology, 81, 141-145. https://doi.org/10.1037/h0027474

Young, A. W., \& Burton, A. M. (2018). Are we face experts? Trends in Cognitive Sciences, 22, 100-110. https://doi.org/10.1016/j.tics.2017.11.007

Young, A. W., Hellawell, D., \& Hay, D. C. (1987). Configural information in face perception. Perception, 16, 747-759. https://doi.org/10.1068/p160747n 
Table 1. Demographics of sample

\begin{tabular}{|c|c|c|c|}
\hline Demographic & & Parkinson's $(n=16)$ & Controls $(n=17)$ \\
\hline Sex & Male (\%) & $10(62.5 \%)$ & $8(47.1 \%)$ \\
\hline Age (years) & Mean (SD) & $70.13(10.38)$ & $67.94(11.31)$ \\
\hline & Range & $47-85$ & $54-88$ \\
\hline PD duration (years) & Mean (SD) & $6.88(5.19)$ years & \\
\hline & Range & $.25-15$ years & \\
\hline Hoehn \& Yahr Stage & I & 3 & \\
\hline & II & 9 & \\
\hline & III & 4 & \\
\hline Mill Hill $(\max =17)$ & Mean (SD) & $10.38(2.09)$ & $10.76(2.2)$ \\
\hline
\end{tabular}


Table 2. Whole face emotion recognition. Mean scores, standard deviations (SD), range of scores, and Mann-Whitney Tests (MWU) comparing groups according to emotion type (Parkinson's (PD; $n=16)$, control $(C ; n=17)$ ) and Wilcoxon signed-ranks analyses (Z-scores and significant $p$ values) comparing participants according to orientation (upright (U), inverted (I)).

\begin{tabular}{|c|c|c|c|c|c|c|c|c|c|c|c|c|}
\hline & \multicolumn{2}{|c|}{$\begin{array}{c}\text { Anger } \\
(\max =10)\end{array}$} & \multirow[t]{2}{*}{$\begin{array}{l}Z \\
p\end{array}$} & \multicolumn{2}{|c|}{$\begin{array}{c}\text { Disgust } \\
\text { (max = 10) }\end{array}$} & \multirow[t]{2}{*}{$\begin{array}{l}Z \\
p\end{array}$} & \multicolumn{2}{|c|}{$\begin{array}{c}\text { Fear } \\
(\max =10)\end{array}$} & \multirow[t]{2}{*}{$\begin{array}{l}Z \\
p\end{array}$} & \multicolumn{2}{|c|}{$\begin{array}{c}\text { Sad } \\
(\max =10)\end{array}$} & \multirow[t]{2}{*}{$\begin{array}{l}Z \\
p\end{array}$} \\
\hline & $U$ & I & & $U$ & I & & $U$ & I & & $U$ & I & \\
\hline$P D$ & 3.63 & 2.25 & -2.38 & 5.31 & 2.31 & -3.01 & 5.69 & 5.06 & -.924 & 6.38 & 4.31 & -3.04 \\
\hline$(n=16)$ & $(2.81)$ & $(1.61)$ & .007 & $(2.09)$ & $(2.50)$ & .001 & $(2.68)$ & $(2.67)$ & - & (3.32) & $(2.65)$ & $<.001$ \\
\hline Range & $1-10$ & $0-6$ & & $2-9$ & $0-9$ & & $1-10$ & $1-10$ & & $0-10$ & $0-9$ & \\
\hline$C$ & 5.12 & 3.12 & -2.41 & 7.18 & 4.18 & -3.54 & 7.59 & 5.47 & -2.15 & 7.24 & 6.41 & -1.39 \\
\hline$(n=17)$ & $(1.97)$ & (1.93) & .007 & $(1.59)$ & (2.19) & $<.001$ & $(1.77)$ & $(2.96)$ & .015 & $(2.80)$ & $(1.91)$ & - \\
\hline Range & $0-8$ & $0-8$ & & $4-10$ & $1-9$ & & $5-10$ & $0-10$ & & $0-10$ & $3-9$ & \\
\hline$M W U$ & 59.5 & 97.5 & & 69.0 & 68.0 & & 81.0 & 123.5 & & 116.5 & 73.5 & \\
\hline$p$ & .005 & - & & .015 & .014 & & .049 & - & & - & .023 & \\
\hline
\end{tabular}


Table 3. Face Composite Paradigm. Mean, standard deviation (SD), and range of scores according to orientation and alignment for PD patients $(n=16)$ and controls $(n=17)$.

\begin{tabular}{|c|c|c|c|c|c|}
\hline \multirow[t]{2}{*}{$\begin{array}{l}\text { Emotion } \\
(\max =12)\end{array}$} & \multirow[t]{2}{*}{ Group } & \multicolumn{2}{|c|}{$\begin{array}{c}\text { Upright } \\
\text { Mean (SD) }\end{array}$} & \multicolumn{2}{|c|}{$\begin{array}{c}\text { Inverted } \\
\text { Mean (SD) }\end{array}$} \\
\hline & & Aligned & Non-aligned & Aligned & Non-aligned \\
\hline \multirow[t]{4}{*}{ Anger } & $P D$ & $4.44(2.34)$ & $4.44(1.97)$ & $4.25(2.60)$ & $3.63(1.89)$ \\
\hline & & $1-10$ & $1-7$ & $0-9$ & $1-7$ \\
\hline & $C$ & $4.53(1.81)$ & $5.65(2.18)$ & $4.35(1.80)$ & $5.35(2.45)$ \\
\hline & & $2-8$ & $2-10$ & $1-9$ & $1-10$ \\
\hline \multirow[t]{4}{*}{ Disgust } & $P D$ & $3.19(1.64)$ & $3.19(1.60)$ & $2.75(1.29)$ & $2.31(1.14)$ \\
\hline & & $0-6$ & $1-7$ & $1-6$ & $0-5$ \\
\hline & $C$ & $3.59(1.66)$ & $4.88(2.45)$ & $2.71(1.72)$ & $2.88(1.45)$ \\
\hline & & $1-7$ & $0-11$ & $0-6$ & $1-6$ \\
\hline \multirow[t]{4}{*}{ Fearful } & $P D$ & $5.38(2.09)$ & $5.75(2.52)$ & $3.81(2.32)$ & $4.56(2.10)$ \\
\hline & & $1-10$ & $1-10$ & $0-8$ & $1-8$ \\
\hline & $C$ & $4.47(2.48)$ & $6.53(1.74)$ & $4.06(2.05)$ & $5.00(1.66)$ \\
\hline & & $1-11$ & $4-10$ & $0-8$ & $2-8$ \\
\hline \multirow[t]{4}{*}{ Sadness } & $P D$ & $3.31(2.18)$ & $4.63(2.39)$ & $2.50(1.55)$ & $2.25(1.69)$ \\
\hline & & $0-8$ & $1-9$ & $0-5$ & $0-6$ \\
\hline & $C$ & $4.65(2.18)$ & $5.06(2.39)$ & $3.00(1.41)$ & $3.65(1.87)$ \\
\hline & & $1-8$ & $3-8$ & $1-6$ & $1-8$ \\
\hline Total & $P D$ & $16.31(4.91)$ & $18.00(5.09)$ & $13.31(4.80)$ & $12.75(4.36)$ \\
\hline \multirow[t]{3}{*}{$(\max =48)$} & & $8-24$ & $9-26$ & $4-23$ & $5-21$ \\
\hline & $C$ & $17.24(5.38)$ & $22.12(5.33)$ & $14.12(3.90)$ & $16.88(4.40)$ \\
\hline & & $10-28$ & $16-34$ & $7-22$ & $10-26$ \\
\hline
\end{tabular}



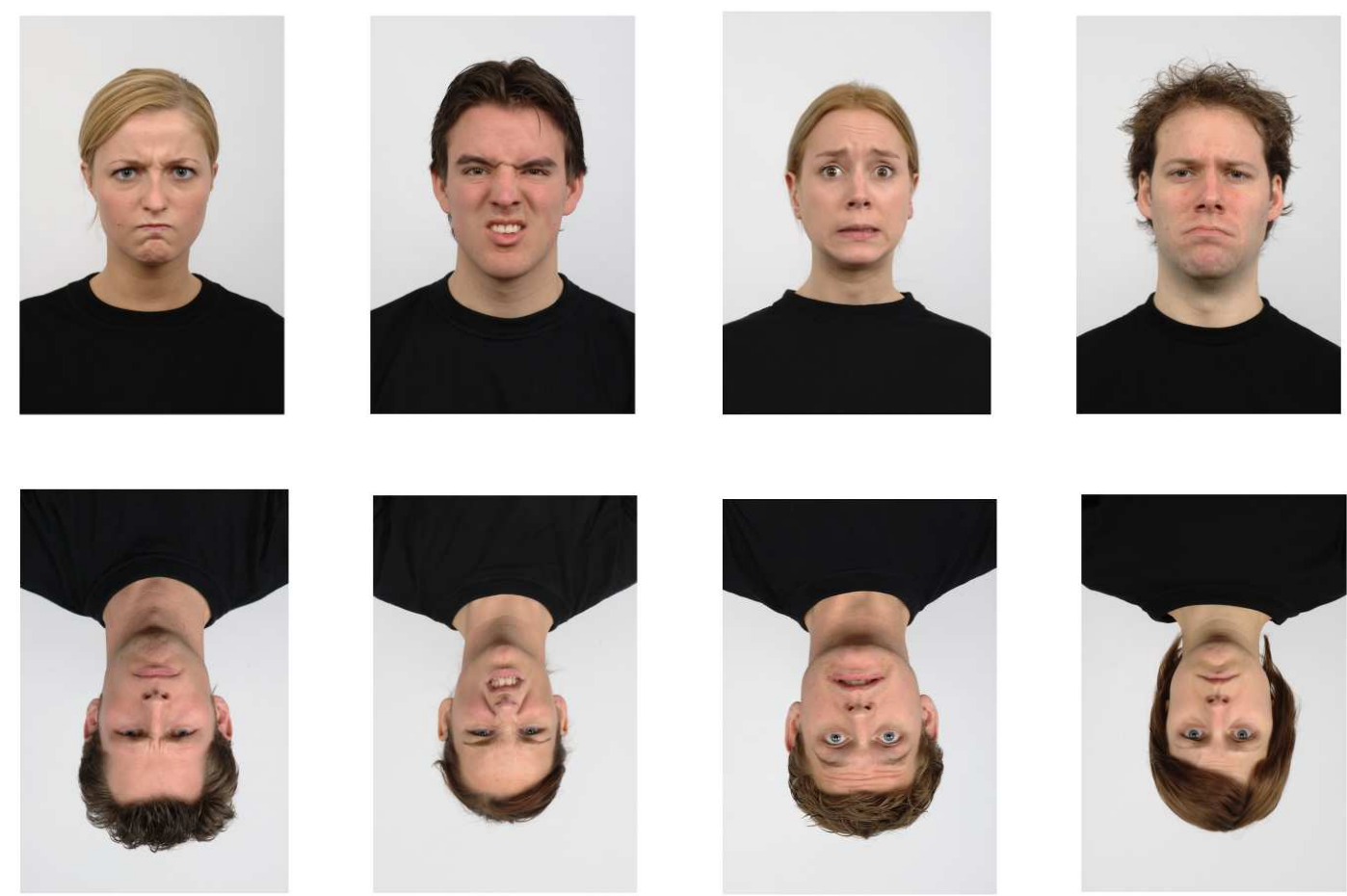

Figure 1. Sample of whole faces upright and inverted for anger, disgust, fear, sadness 

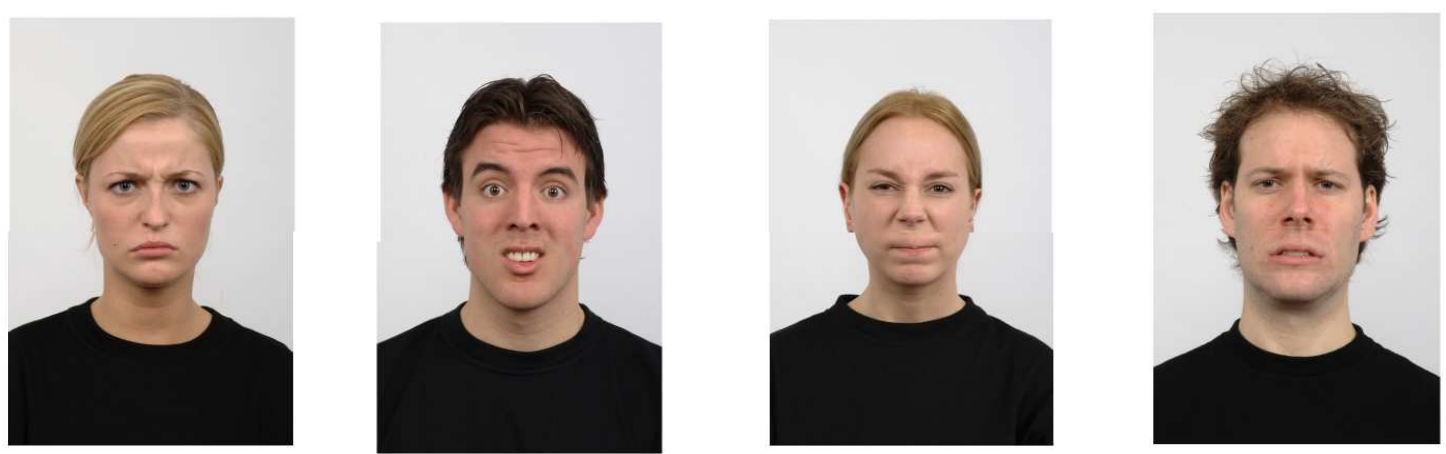

Figure 2. Sample of upright aligned composite faces (Anger/Sadness; Fear/Disgust; Disgust/Anger; Sadness/Fear).
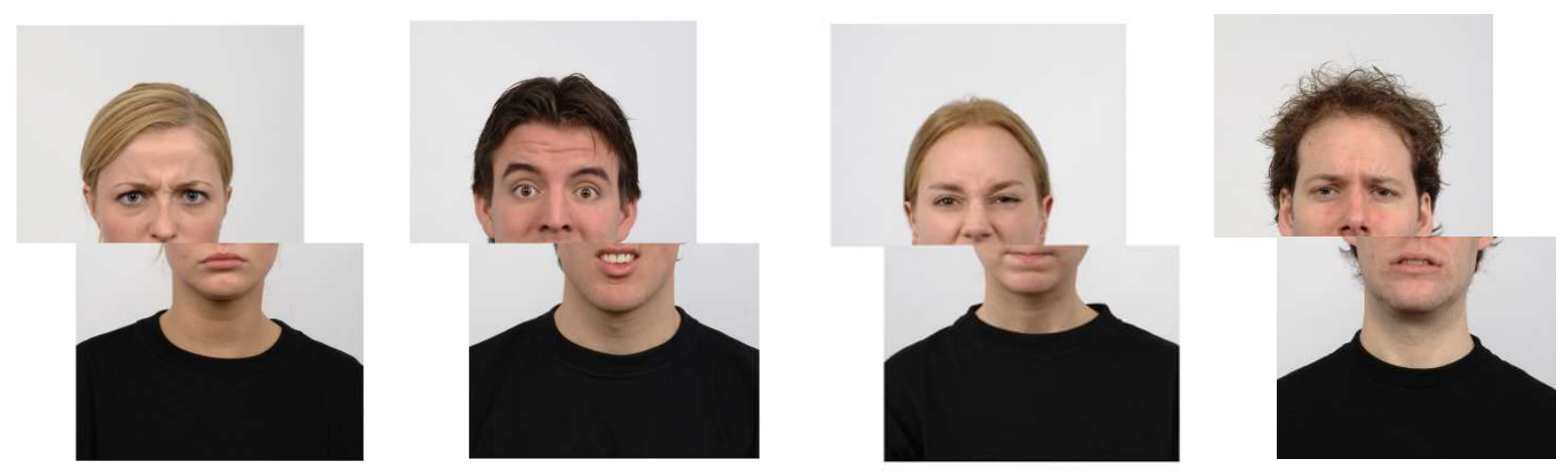

Figure 3. Sample of upright non-aligned composite faces (Anger/Sadness;

Fear/Disgust; Disgust/Anger; Sadness/Fear). 